\title{
Quelles perspectives de recherche en productions animales?
}

\author{
Patrick Herpin \\ Directeur scientifique animal \\ et produits animaux \\ Inra \\ 147, rue de l'Université \\ 75338 Paris cedex 07 \\ France \\ <Patrick.Herpin@rennes.inra.fr>
}

es recherches en productions animales de demain devront, tout à la fois, produire des données génériques pour la connaissance du vivant, et proposer des systèmes de production innovants et compétitifs, en rupture avec l'existant, en s'accompagnant d'une large ouverture disciplinaire et partenariale.

Après avoir été l'un des acteurs majeurs de la modernisation de l'élevage après guerre, la recherche agronomique des 20 dernières années a largement contribué à l'essor du secteur agroalimentaire français, en intégrant les innovations provenant des avancées très importantes dans le domaine des sciences du vivant. Aujourd'hui, plusieurs grands enjeux sont venus replacer l'agronomie et les productions animales au cour des débats de société : la prise de conscience de la nécessité de se situer dans une perspective de développement durable pour conserver et transmettre notre patrimoine environnemental aux futures générations ; la mondialisation de l'économie qui impose une concurrence exacerbée entre grandes régions de production; la dimension planétaire des problématiques (changement climatique, gestion de la biodiversité, qualité de l'eau, qualité et sécurité de l'alimentation, maladies émergentes, bioénergies) ; la nécessité d'accroître encore à l'avenir l'offre alimentaire mondiale pour répondre à une démographie galopante ; et l'évolution du statut de l'animal qui questionne à la fois les méthodes d'expérimentation animale et les pratiques d'élevage.

Pour répondre à ces enjeux, les recherches en productions animales de demain devront, tout à la fois, produire des données génériques pour la connaissance du vivant et proposer des systèmes de production innovants et compétitifs, en rupture avec l'existant, en s'accompagnant d'une large ouverture disciplinaire et partenariale avec l'ensemble des organismes concernés par le domaine des productions et de la santé animales.

Les relations entre l'élevage, ses produits et l'environnement devront être explorées, analysées, modélisées pour faire évoluer nos pratiques et tenter de réconcilier élevage et écologie.

Les préoccupations sur les impacts environnementaux de l'élevage sont fondées au niveau planétaire, et le monde de l'élevage doit résolument s'engager sur la problématique du développement durable. Grâce aux premiers travaux engagés, une partie des solutions techniques existe déjà pour réduire les émissions de méthane et/ou de nitrates, pour gérer les ressources en eau, pour maintenir la biodiversité ou pour mâttriser l'usage raisonné des anti-infectieux ou des antiparasitaires. Il faut poursuivre dans ce sens, et aller plus loin en intégrant aussi la production d'aménités environnementales par l'élevage (contribution à la biodiversité, séquestration de carbone, production d'énergie ou de fertilisants à partir des effluents, valorisation de coproduits issus des filières bioénergétiques, entretien des territoires, etc.) et les interrogations sur la production et l'utilisation de l'énergie. Réciproquement, il nous faut analyser comment l'élevage pourra s'adapter au nouveau contexte du changement climatique (raréfaction de la ressource en eau, modifications de la flore prairiale, diffusion de nouveaux vecteurs arthropodes de maladies animales, élévation de la température de l'eau et de l'air, etc.). Tout cela nécessite probablement une "remise à plat " des façons de produire, et les biologistes animaux devront, plus que par le passé, jouer un rôle moteur et central dans les discussions et 
travaux à venir pour engager les ruptures nécessaires.

Pour y parvenir, il convient, sans négliger les recherches disciplinaires, de privilégier les démarches interdisciplinaires, globales et systémiques.

Les produits animaux : quelle place dans l'alimentation de l'bomme demain? Il convient de mener une réflexion large et prospective sur l'évolution de la place des produits animaux dans l'alimentation de l'homme, en prenant en compte l'ensemble des enjeux qui lui sont liés, qu'ils soient de nature économique, sociologique, nutritionnelle, zootechnique, environnementale ou sanitaire. Cette réflexion nous conduira à revisiter éventuellement nos programmes, priorités et dispositifs, à promouvoir de nouveaux projets et de nouvelles synergies, à nous interroger sur la manière de produire. La construction de la qualité nutritionnelle et organoleptique des produits animaux, au sein d'un régime alimentaire équilibré, leur qualité sanitaire en relation avec l'émergence de nouveaux modes d'élevage moins confinés devront faire l'objet d'une attention particulière, tout comme le transfert de xénobiotiques et de micropolluants au sein de la chaîne alimentaire. Cette notion de chaîne alimentaire devra être déclinée dans des programmes collaboratifs interdisciplinaires, la qualité des produits se construisant dès l'amont, au sein des systèmes d'élevage. Il faudra, par ailleurs, décrire les liens complexes entre alimentation et environnement, " l'alimentation durable ", un champ de recherche relativement récent où la production d'indicateurs fiables de type écobilans et analyse de cycle de vie (de la production à la consommation) reste délicate.

Le formidable potentiel de progrès et d'innovation offert par la génomique et la postgénomique devra être exploré.

Dans ce domaine, il convient de renforcer les recherches sur les mécanismes de régulation de l'expression des gènes (variabilité génétique, empreinte, épigénétique, contrôle de la traduction) et d'organiser le génotypage à haut et moyen débit. Il faudra aussi prévoir la transposition des acquis de la recherche obtenus dans les espèces majeures aux productions dites "secondaires" (caprins, ovins, dinde, pintade, canard, poissons autres que salmonidés) dont le génome n'est pas connu (cartographie, approches comparatives). Il est aussi évident que pour valoriser les possibilités d'investigation offertes par les approches génomiques, structurales ou fonctionnelles, il est nécessaire de disposer de phénotypes les plus fins et précis possibles correspondant aux caractères d'intérêt et aux fonctions biologiques sous-jacentes. Que l'on parle de phénotypage haut débit, de phénotypage fin ou de multiphénotypage, l'ambition est bien de disposer de mesures sur plusieurs caractères élémentaires pour un caractère synthétique d'intérêt, et de mesures de plusieurs caractères pour un même animal. Couplée aux avancées dans le domaine de la bio-informatique et de la modélisation, cette dynamique ouvrira des perspectives ambitieuses d'intégration des connaissances vers la biologie prédictive et synthétique. Cette connaissance approfondie des génomes aura des implications considérables en matière de sélection - la sélection génomique - qu'il conviendra d'explorer, d'anticiper et de diffuser. Il est aussi attendu des génomiciens animaux qu'ils s'approprient les nouveaux enjeux du développement durable et investissent notamment le domaine des relations entre élevage et environnement (respect de l'environnement, adaptation au changement climatique, robustesse et adaptabilité des animaux, etc.).

La maîtrise des processus infectieux, émergents ou zoonotiques, nécessitera la mise ouvre d'une véritable écologie des maladies, intégrant la dimension environnementale. La pathologie des différentes espèces d'animaux de rente reste dominée par les processus infectieux, émergents ou récurrents. Les événements sanitaires récents (maladies à prions, fièvre aphteuse, syndrome respiratoire aigu sévère (SRAS), influenza aviaire, fièvre catarrhale ovine) ont replacé l'émergence et la réémergence des maladies infectieuses des animaux au cour des nouvelles questions de santé animale et de santé publique vétérinaire. De nombreux facteurs favorables aux émergences se combinent actuellement. La maîtrise des maladies animales et la sécurité des produits qui en résulte constituent la première priorité des filières animales et des gestionnaires du risque, d'autant que l'évolution réglementaire européenne repousse vers l'amont la responsabilité de la qualité sanitaire des produits. Ces nouveaux défis posent de nombreuses questions scientifiques qui relèvent d'approches disciplinaires et intégratives et d'une véritable écologie des maladies infectieuses, intégrant la dimension environnementale.
Analyser et accompagner l'évolution du statut de l'animal dans la société.

L'évolution du statut de l'animal d'élevage dans la société interfère à la fois avec l'expérimentation animale, les activités d'élevage et l'acceptabilité des produits animaux. Au carrefour de considérations socioculturelles, biologiques, zootechniques et morales, la définition du bienêtre animal est d'ailleurs loin d'être unanimement partagée par les différentes communautés de pensée. Cette dimension continuera à être revisitée, enrichie, explicitée, notamment par la mise en œuvre d'approches pluridisciplinaires et d'une évaluation globale, incluant l'état de santé de l'animal. Elle sera prise en compte dans l'élaboration des systèmes d'élevage, sans toutefois tomber dans l'excès et sans perdre de vue que l'amélioration du bien-être animal doit remettre l'éleveur au coeur du système de production. Les techniques de clonage et de transgenèse animale sont porteuses de progrès scientifique et d'innovations considérables, mais suscitent déjà des interrogations légitimes sur leur utilité et leur danger potentiel. Il faut donc simultanément poursuivre la construction de la position de l'Inra en matière de réflexion éthique et d'acceptabilité sociale (évaluation des attentes sociétales, dialogue avec la société), et engager les recherches nécessaires pour consolider notre expertise scientifique. Des progrès sont attendus dans le domaine de la production de connaissances, de l'innovation thérapeutique, ou enfin de l'innovation agronomique.

Pour s'approprier pleinement ces différentes dimensions, les chercheurs en sciences animales devront résolument ouvrir leurs réflexions et leurs projets à d'autres disciplines (écologie, agronomie, nutrition humaine, sociologie, économie de l'élevage et des produits, etc.) et construire leurs questions de recherche en diversifiant leurs partenariats, dans le cadre d'un dialogue renouvelé et constructif avec la société. Le rapprochement annoncé et souhaité entre la recherche et la formation dans le domaine agronomique et vétérinaire sera un sérieux atout pour atteindre ces objectifs et permettra d'améliorer la performance du dispositif, de renforcer sa visibilité, sa reconnaissance, son attractivité et ses capacités d'action à l'international, et enfin de mobiliser de nouvelles compétences scientifiques autour de ces grands enjeux. 